\title{
Higher Dispositional Optimism Predicts Better Health-Related Quality of Life After Esophageal Cancer Surgery: A Nationwide Population-Based Longitudinal Study
}

\author{
Yangjun Liu, MD', Erik Pettersson, $\mathbf{P h D}^{2}$, Anna Schandl, $\mathbf{P h D}{ }^{1,3}$, Sheraz Markar, $\mathbf{P h D}^{1,4}$, \\ Asif Johar, MSc ${ }^{1}$, and Pernilla Lagergren, $\mathbf{P h D}^{1,4}$ \\ ${ }^{1}$ Department of Molecular Medicine and Surgery, Karolinska Institutet, Karolinska University Hospital, Stockholm, \\ Sweden; ${ }^{2}$ Department of Medical Epidemiology and Biostatistics, Karolinska Institutet, Stockholm, Sweden; ${ }^{3}$ Department \\ of Anesthesiology and Intensive Care, Södersjukhuset, Stockholm, Sweden; ${ }^{4}$ Department of Surgery and Cancer, Imperial \\ College London, London, UK
}

\begin{abstract}
Purpose. To assess whether higher dispositional optimism could predict better health-related quality of life (HRQL) after esophageal cancer surgery.

Methods. This Swedish nationwide longitudinal study included 192 patients who underwent esophagectomy for cancer. The exposure was dispositional optimism measured by the Life Orientation Test-Revised (LOT-R) at 1 year post-surgery. Patients were categorized into four subgroups (very low, moderately low, moderately high, and very high dispositional optimism) based on the quartile of the LOT-R sum score. The outcome was HRQL assessed by the European Organization for Research and Treatment of Cancer (EORTC) Quality of Life Questionnaire-Core 30 (QLQ-C30) and Quality of Life Questionnaire-EsophagoGastric module 25 (QLQ-OG25) at 1, 1.5, and 2 years postsurgery. Linear mixed-effects models, adjusted for potential confounders, were used to examine the mean score difference (MSD) with 95\% confidence interval of HRQL among the four patient subgroups.
\end{abstract}

Supplementary Information The online version contains supplementary material available at https://doi.org/10.1245/s10434021-10026-w.

(C) The Author(s) 2021

First Received: 5 January 2021

Accepted: 26 March 2021;

Published Online: 19 April 2021

$\mathrm{P}$. Lagergren, $\mathrm{PhD}$

e-mail: pernilla.lagergren@ki.se
Results. Patients with very high dispositional optimism reported clinically relevantly better global quality of life, emotional function, and social function (MSD range 10-16) and less severe symptoms in pain, dyspnea, diarrhea, eating difficulty, anxiety, dry mouth, trouble with taste, worry about weight loss, and self-doubt about body image (MSD range -9 to -22 ) than patients with lower dispositional optimism. Patients with moderately high dispositional optimism reported clinically and statistically significantly better global quality of life (MSD 10) and less severe diarrhea (MSD - 9) than patients with lower dispositional optimism. Adjusted MSDs were constant over the three time points in all aspects except for eating difficulty.

Conclusions. Measuring dispositional optimism could help identify patients at higher risk of poor HRQL recovery after esophageal cancer surgery.

Esophageal cancer is a malignant tumor that ranks as the sixth leading cause of cancer death globally. ${ }^{1}$ Esophagectomy, often in combination with chemotherapy or chemoradiotherapy, is the main treatment approach with curative intent. Patients with esophagectomy for cancer usually suffer from substantially decreased health-related quality of life (HRQL), especially within 6 months postsurgery. ${ }^{2,3}$ From 1 year post-surgery, HRQL is reported to have recovered to a large extent. ${ }^{2,3}$ Previous studies have demonstrated that histology type, tumor stage, tumor location, operation approach, postoperative complications, and comorbidity are predictors of poor postoperative HRQL. ${ }^{4-7}$ However, patients with similar characteristics in 
these aspects still report varied HRQL, indicating that other factors, such as personality traits, might also have an influence.

Dispositional optimism is a relatively stable personality trait, which refers to the global expectation that more desirable than bad things will happen in the future. ${ }^{8}$ No previous study has assessed the association between dispositional optimism and HRQL among patients with esophageal cancer. Studies conducted among patients with other subtypes of cancer have shown that higher dispositional optimism is associated with better HRQL in several aspects, such as global quality of life, emotional function, social function, pain, and body image. ${ }^{9-15}$ However, its associations with other aspects including physical function, role function, and cognitive function were ambiguous, as some studies reported significant associations while others reported nonsignificant associations. ${ }^{12-14,16}$ In addition, one study found that the association between dispositional optimism and HRQL tends to attenuate when near death. ${ }^{17}$ Given that more than $50 \%$ of surgically treated patients with esophageal cancer die within 5 years post-surgery, ${ }^{18}$ it remains uncertain whether higher dispositional optimism could predict better HRQL in this population. Clarifying this predictive effect may help identify vulnerable patients who are at higher risk of suffering from poor HRQL after surgery, thus providing early and personalized interventions to patients in need. Moreover, as dispositional optimism can be increased via psychological interventions, ${ }^{19}$ if this predictive effect exists, it may also imply a potential intervention target to improve postoperative HRQL.

In this study, we aimed to use Swedish nationwide population-based longitudinal data to assess whether higher dispositional optimism predicts better HRQL after esophageal cancer surgery.

\section{PATIENTS AND METHODS}

\section{Study Design and Data Collection}

Data for this longitudinal study were drawn from a prospective, ongoing Swedish nationwide cohort study entitled Oesophageal Surgery on Cancer patients-Adaptation and Recovery (OSCAR). Detailed description of the OSCAR study has been presented elsewhere. ${ }^{20}$ In brief, it includes 1-year esophageal cancer survivors without cognitive dysfunction who underwent curative-intent esophagectomy in Sweden from 1 January 2013 onwards (response rate around $66 \%$ ). ${ }^{20}$ Eligible patients are identified through collaboration with pathology departments at eight hospitals performing esophagectomy in Sweden. ${ }^{20}$ Survival information is collected through linkage to the
Swedish Register of the Total Population and the Swedish cause of death register. ${ }^{20}$ Follow-ups on patient-reported outcomes start from 1 year and last until 5 years postsurgery through personal interview or mailing paper questionnaires. $^{20}$ In addition, patients' demographics are retrieved from the Swedish national health data registries and the Swedish Longitudinal Integration Database for Health Insurance and Labor Market Studies. ${ }^{20}$ Clinical data are obtained from medical charts, the Swedish Patient Registry, and the Swedish Cancer Registry. ${ }^{20}$ The study was approved by the Regional Ethical Review Board in Stockholm, Sweden (diary number 2013/844-31/1), and written consent was obtained from all participants before inclusion.

The present study included patients who underwent esophagectomy for cancer between 1 January 2013 and 28 February 2018, and incorporated three follow-up time points at $1,1.5$, and 2 years post-surgery. Patients who died during the follow-up period, had psychiatric history, or were diagnosed with noncancerous neoplasm (dysplasia) were excluded.

\section{Exposure: Dispositional Optimism}

Dispositional optimism was measured at 1 year postsurgery using the Swedish version of Life Orientation TestRevised (LOT-R). ${ }^{21,22}$ LOT-R comprises three positively worded items and three negatively worded items, ${ }^{21,22}$ and asks patients to report their agreement with each item on a five-point Likert scale ranging from 0 ("strongly disagree") to 4 ("strongly agree"). ${ }^{22}$

Due to the ambiguous dimensionality of LOT-R and absence of psychometric study in patients with esophageal cancer, we conducted a series of confirmatory factor analyses to assess the factor structure of LOT-R. Detailed results can be found elsewhere. ${ }^{23}$ Because the first negatively worded item had negative loading in the best-fit model and its response distribution was bimodal, we removed this item and adopted the model assuming one factor (dispositional optimism) with correlated errors between the two reversed negatively worded items. The internal reliability estimated by McDonald's omega for this model was 0.49 [95\% bootstrapped confidence interval (CI) $0.31-0.62]$.

The remaining five items of LOT-R were summed, of which the two negatively worded items were reversed. A higher sum score represents higher dispositional optimism. Based on the quartile of the sum score, patients were categorized into four subgroups with very low, moderately low, moderately high, and very high dispositional optimism. 


\section{Outcome: $H R Q L$}

HRQL was measured at $1,1.5$, and 2 years post-surgery using the European Organization for Research and Treatment of Cancer Quality of Life Questionnaire-Core 30 (EORTC QLQ-C30) and disease site-specific (EsophagoGastric) module EORTC QLQ-OG25. ${ }^{24,25}$ Both questionnaires are validated in Swedish and have demonstrated good psychometric properties. ${ }^{24,25}$

The EORTC QLQ-C30 contains 30 items with Likert scaling and measures HRQL aspects of cancer patients in general. It consists of one global quality-of-life subscale, five functional subscales (physical, role, emotional, social, and cognitive), three symptom subscales (fatigue, pain, and nausea/vomiting), and six single items (dyspnea, appetite loss, insomnia, constipation, diarrhea, and financial difficulty). ${ }^{24}$ Items in the global quality-of-life subscale range from 1 ("very poor") to 7 ("excellent"), while items in other subscales score from 1 ("not at all") to 4 ("very much"). ${ }^{24}$

The EORTC QLQ-OG25 is a 25-item esophagogastric cancer-specific questionnaire, which comprises six symptom subscales (dysphagia, eating difficulty, reflux, odynophagia, pain and discomfort, and anxiety) and ten single items (eating in front of others, dry mouth, trouble with taste, trouble swallowing saliva, choked when swallowing, trouble with coughing, trouble talking, weight loss, body image, and hair loss). ${ }^{25}$ All items are scored on a four-point Likert scale ranging from 1 ("not at all") to 4 ("very much"). ${ }^{25}$

Missing values in both questionnaires were handled according to the EORTC Scoring Manual, ${ }^{26}$ and the raw score of each HRQL subscale was transformed to a linear scale of $0-100{ }^{26} \mathrm{~A}$ higher score represents better function/global quality of life or higher symptom burden. ${ }^{26} \mathrm{In}$ addition, a single summary score for the EORTC QLQ-C30 was calculated according to the EORTC guideline. ${ }^{27}$

\section{Statistical Analysis}

Sociodemographic and clinical characteristics of all included patients as well as the four patient subgroups were summarized. We compared the overall mean of the LOT-R sum score between patients with different sociodemographic and clinical characteristics using $t$-test or analysis of variance (ANOVA). Linear mixed-effects model was used to examine the mean score differences (MSDs) of HRQL among the four patient subgroups with hierarchical dispositional optimism levels. Covariates include time (1, 1.5 , and 2 years post-surgery), age (continuous variable), sex (female or male), cohabitation status (non-cohabitating or cohabitating), education level (9-year compulsory school, upper secondary school, or higher education),
Charlson Comorbidity Index ${ }^{28}(0$ or $\geq 1)$, tumor stage (complete regress after neoadjuvant therapy/I, II, or IIIIV), histology (squamous cell carcinoma or adenocarcinoma), postoperative Clavien-Dindo complication score (none, I-II, or III-IV), and weight change after surgery (continuous variable). Sociodemographic factors were included because they are potential confounders associated with both dispositional optimism and HRQL. ${ }^{29,30}$ Although no previous studies have suggested that clinical factors affect dispositional optimism, we included these factors as covariates to increase the estimation precision because they are strongly associated with postoperative HRQL. ${ }^{4-7,31}$ In addition, the interaction effect between dispositional optimism and time was examined using the Wald test. In all models, four covariance matrices (unstructured, independent, exchangeable, and identity) were compared using the Bayesian information criterion (BIC), and the one with lower BIC is preferred because it indicates better balance between goodness of fit and parsimony. ${ }^{32}$

HRQL measures several aspects; to minimize the type I error due to multiple comparisons, we tested the statistical significance of adjusted MSD only if it had clinical relevance based on evidence-based guidelines. ${ }^{33-36}$ The adjusted MSD of HRQL aspects measured by EORTC QLQ-C30 has four grades: trivial (circumstances unlikely to have any clinical relevance or where there was no difference), small (subtle but clinically relevant), medium (likely to be clinically relevant but to a lesser extent), or large (of unequivocal clinical relevance). ${ }^{33,34} \mathrm{We}$ regarded medium and large differences as clinically relevant in the present study. For other HRQL aspects, an adjusted MSD of $\geq 10$ was considered clinically relevant. ${ }^{35,36}$

Stata 13 and SAS 9.4 were used for the statistical analyses. All 95\% confidence intervals (CIs) were two sided.

\section{RESULTS}

\section{Study Participants}

Figure 1 displays the detailed process of patient selection. Between 1 January 2013 and 28 February 2018, 647 patients underwent curative-intent esophageal cancer surgery in Sweden. Of these, 407 patients were invited to participate in the study, and $265(65 \%)$ patients consented to participation and finished the first (1-year) interview. Nonparticipation was mainly due to unwillingness, poor health, and cancer recurrence. ${ }^{20}$ In addition, 73 patients were further excluded due to death during follow-up ( $n=$ 49), psychiatric history before surgery $(n=5)$, noncancerous histology $(n=3)$, and lack of essential data $(n=$ 16), leaving 192 patients included in the analysis. Among 
FIG. 1 Flowchart of patient selection for inclusion. OSCAR study Oesophageal Surgery on Cancer patients-Adaptation and Recovery (OSCAR), a prospective, ongoing Swedishnationwide cohort study, LOT-R Life Orientation Test-Revised

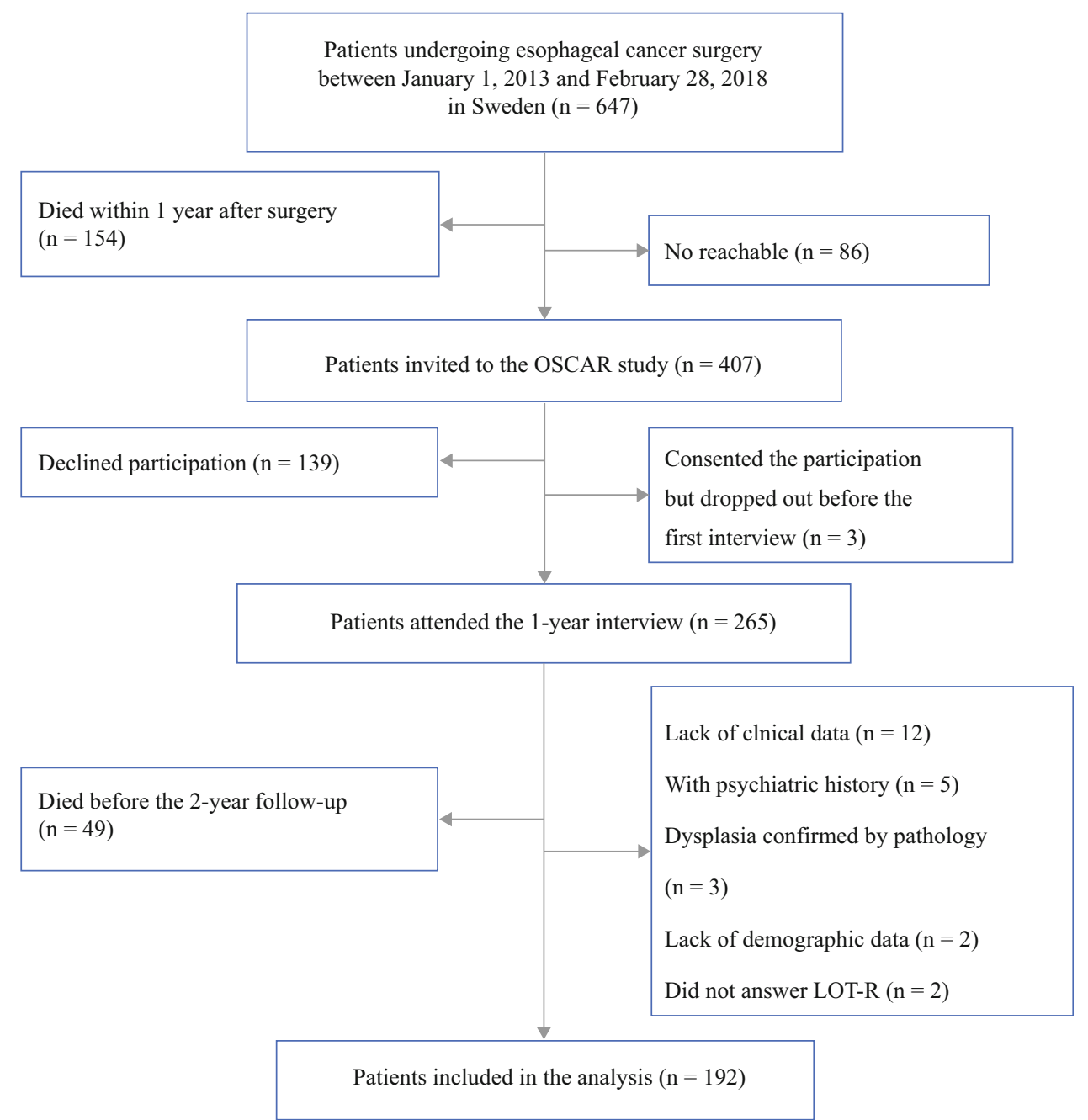

them, 170 and 156 patients answered the 1.5- and 2-year questionnaire, respectively.

\section{Characteristics of Participants}

Table 1 presents the sociodemographic and clinical characteristics of the 192 patients. Mean age at surgery was 66.3 years [standard deviation (SD) 8.5 years, range 38.2-83.7 years). Most patients were male (85.4\%) and married/cohabitating $(77.1 \%)$. The mean of the LOT-R sum score was 15.2 (SD 3.0) with range of 6-20. Based on the quartile of LOT-R sum score, patients were categorized into four subgroups with hierarchical dispositional optimism levels: very low $(n=48$, LOT-R sum score range 6-13), moderately low ( $n=51$, LOT-R sum score range 14-15), moderately high ( $n=45$, LOT-R sum score range $16-17)$, and very high ( $n=48$, LOT-R sum score range 19-20). Sociodemographic and clinical characteristics of the four patient subgroups are presented in Table 1. Patients with different sociodemographic and clinical characteristics reported similar LOT-sum scores (Supplementary Table S1).

\section{Time-Invariant Predictive Effect of Dispositional Optimism on HRQL}

In almost all HRQL aspects except for eating difficulty, the predictive effect of dispositional optimism on HRQL was not modified by time. Over the three assessment time points, there was no clinically relevant difference between patients with very low and moderately low dispositional optimism in any HRQL aspect (Supplementary Table S2). However, compared with patients with very low and moderately low dispositional optimism, patients with moderately high dispositional optimism reported clinically relevantly and statistically significantly better global quality of life (MSD 10, 95\% CI 4-17) and less severe diarrhea (MSD -9, 95\% CI - 18 to - 1; Fig. 2; Supplementary Table S2). Compared with patients with very low, moderately low, and moderately high dispositional 
TABLE 1 Characteristics of all included patients with esophagectomy for cancer and four patient subgroups with hierarchical dispositional optimism levels

$$
\begin{aligned}
& \text { All included patients } \\
& (n=192)
\end{aligned}
$$

\begin{tabular}{|c|c|c|c|c|c|}
\hline & \multirow{2}{*}{$\begin{array}{l}\text { All included patients } \\
(n=192)\end{array}$} & \multicolumn{4}{|c|}{ Dispositional optimism level } \\
\hline & & $\begin{array}{l}\text { Very low }(n= \\
48)\end{array}$ & $\begin{array}{l}\text { Moderately low } \\
(n=51)\end{array}$ & $\begin{array}{l}\text { Moderately high } \\
(n=45)\end{array}$ & $\begin{array}{l}\text { Very high }(n= \\
\text { 48) }\end{array}$ \\
\hline \multicolumn{6}{|l|}{ LOT-R sum score } \\
\hline Mean \pm SD & $15.2 \pm 3.0$ & $11.3 \pm 1.6$ & $14.5 \pm 0.5$ & $16.5 \pm 0.5$ & $18.9 \pm 0.9$ \\
\hline Range & {$[6,20]$} & {$[6,13]$} & {$[14,15]$} & {$[16,17]$} & {$[18,20]$} \\
\hline \multicolumn{6}{|l|}{ Age (years) } \\
\hline Mean $\pm \mathrm{SD}$ & $66.3 \pm 8.5$ & $64.2 \pm 9.9$ & $67.9 \pm 8.3$ & $65.7 \pm 7.9$ & $67.2 \pm 7.3$ \\
\hline Range & {$[38.2,83.7]$} & {$[38.2,82.0]$} & {$[41.5,83.7]$} & {$[49.9,80.5]$} & {$[50.0,80.0]$} \\
\hline \multicolumn{6}{|l|}{ Sex } \\
\hline Female & $28(14.6)$ & $4(8.3)$ & $7(13.7)$ & $7(15.6)$ & $10(20.8)$ \\
\hline Male & $164(85.4)$ & $44(91.7)$ & $44(86.3)$ & $38(84.4)$ & $38(79.2)$ \\
\hline \multicolumn{6}{|l|}{ Cohabitation status } \\
\hline Non-cohabitating & $44(22.9)$ & $13(27.1)$ & $13(25.5)$ & $10(22.2)$ & $8(16.7)$ \\
\hline Cohabitating & $148(77.1)$ & $35(72.9)$ & $38(74.5)$ & $35(77.8)$ & $40(83.3)$ \\
\hline \multicolumn{6}{|l|}{ Education level } \\
\hline Nine-year compulsory school & $48(25.0)$ & $11(22.9)$ & $16(31.4)$ & $11(24.4)$ & $10(20.8)$ \\
\hline Upper secondary school & $85(44.3)$ & $24(50.0)$ & $19(37.3)$ & $22(48.9)$ & $20(41.7)$ \\
\hline Higher education & $59(30.7)$ & $13(27.1)$ & $16(31.4)$ & $12(26.7)$ & $18(37.5)$ \\
\hline \multicolumn{6}{|l|}{ Neoadjuvant therapy } \\
\hline Yes & $158(82.3)$ & $41(85.4)$ & $38(74.5)$ & $37(82.2)$ & $42(87.5)$ \\
\hline No & $34(17.7)$ & $7(14.6)$ & $13(25.5)$ & $8(17.8)$ & $6(12.5)$ \\
\hline \multicolumn{6}{|l|}{ Operation approach } \\
\hline $\begin{array}{l}\text { Total minimally invasive } \\
\text { esophagectomy }\end{array}$ & $52(27.1)$ & $18(37.5)$ & $11(21.6)$ & $7(15.6)$ & $16(33.3)$ \\
\hline $\begin{array}{l}\text { Hybrid minimally invasive } \\
\text { esophagectomy }\end{array}$ & $63(32.8)$ & $18(37.5)$ & $12(23.5)$ & $17(37.8)$ & $16(33.3)$ \\
\hline Open esophagectomy & $77(40.1)$ & $12(25.0)$ & $28(54.9)$ & $21(46.7)$ & $16(33.3)$ \\
\hline \multicolumn{6}{|l|}{ Tumor stage } \\
\hline $\begin{array}{l}\text { Complete regression after } \\
\text { neoadjuvant therapy or I }\end{array}$ & $71(37.0)$ & $15(31.3)$ & $27(52.9)$ & $12(26.7)$ & $17(35.4)$ \\
\hline II & $62(32.3)$ & $20(41.7)$ & $11(21.6)$ & $16(35.6)$ & $15(31.3)$ \\
\hline III-IV & $59(30.7)$ & $13(27.1)$ & $13(25.5)$ & $17(37.8)$ & $16(33.3)$ \\
\hline \multicolumn{6}{|l|}{ Tumor histology } \\
\hline Adenocarcinoma & $163(84.9)$ & $43(89.6)$ & $39(76.5)$ & $43(95.6)$ & $38(79.2)$ \\
\hline Squamous cell carcinoma & $29(15.1)$ & $5(10.4)$ & $12(23.5)$ & $2(4.4)$ & $10(20.8)$ \\
\hline \multicolumn{6}{|c|}{ Postoperative complications (Clavien-Dindo grade) } \\
\hline No complications & $69(35.9)$ & $21(43.8)$ & $15(29.4)$ & $17(37.8)$ & $16(33.3)$ \\
\hline I-II & $54(28.1)$ & $12(25.0)$ & $18(35.3)$ & $14(31.1)$ & $10(20.8)$ \\
\hline III-IV & $69(35.9)$ & $15(31.3)$ & $18(35.3)$ & $14(31.1)$ & $22(45.8)$ \\
\hline \multicolumn{6}{|l|}{ Charlson comorbidity index } \\
\hline 0 & $94(49.0)$ & $18(37.5)$ & $25(49.0)$ & $27(60.0)$ & $24(50.0)$ \\
\hline 1 & $60(31.3)$ & $19(39.6)$ & $16(31.4)$ & $11(24.4)$ & $14(29.2)$ \\
\hline$\geq 2$ & $38(19.8)$ & $11(22.9)$ & $10(19.6)$ & $7(15.6)$ & $10(20.8)$ \\
\hline
\end{tabular}

All values are $n(\%)$ unless otherwise stated, and the percentage is rounded up, which in some cases gives a sum not equaling to $100 \%$ $L O T-R$ life orientation test-revised 
Functional aspects of EORTC QLQ-C30

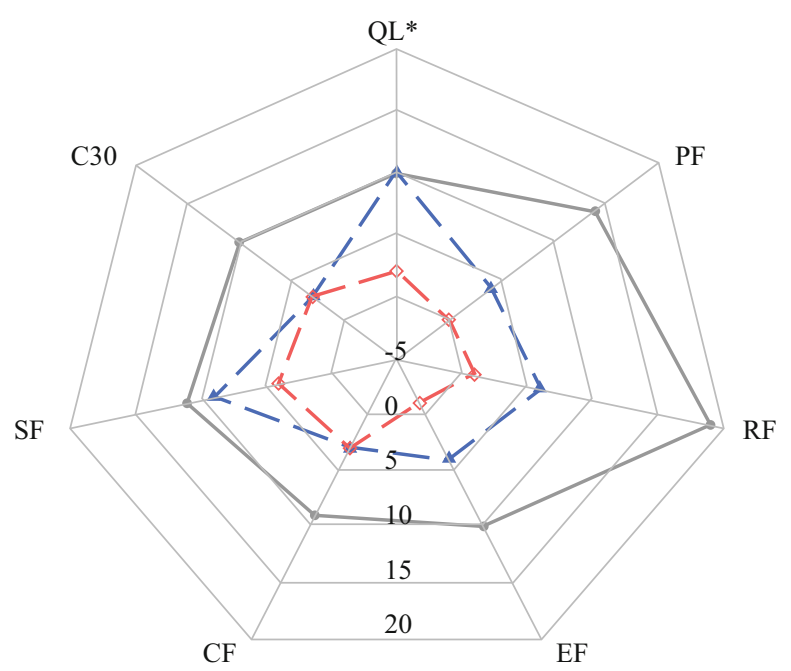

Apects of EORTC QLQ-OG25

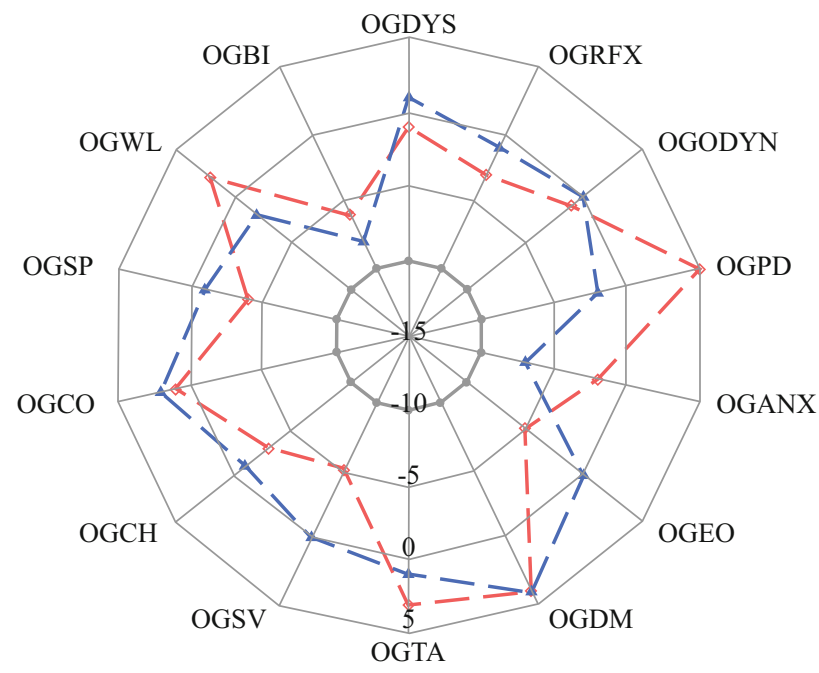

FIG. 2 Mean score differences in health-related quality of life aspects between patients with moderately high dispositional optimism and lower (very low/moderately low) dispositional optimism over the three assessment time points $(1,1.5$, and 2 years after esophageal cancer surgery). EORTC QLQ-C3O European Organization for Research and Treatment of Cancer Quality of Life QuestionnaireCore 30, $Q L$ global quality of life, $P F$ physical function, $R F$ role function, $E F$ emotional function, $C F$ cognitive function, $S F$ social function, C30 EORTC QLQ-C30 summary score, $F A$ fatigue, $N V$ nausea/vomiting, $P A$ pain, $D Y$ dyspnea, $S L$ insomnia, $A P$ appetite

optimism, patients with very high dispositional optimism reported clinically relevantly better global quality of life, emotional function, and social function (MSD range 10-16; Fig. 3; Supplementary Table S2) and less severe symptoms of pain, dyspnea, diarrhea, anxiety, dry mouth, trouble with taste, worry about weight loss, and self-doubt
Symptom aspects of EORTC QLQ-C30

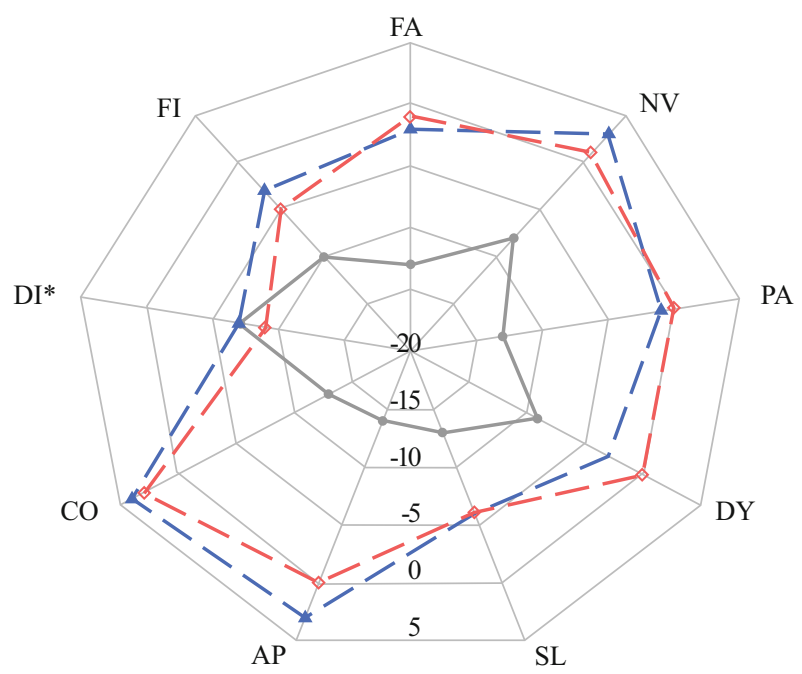

Mean score diffference between patients

with different dispositional optimism level

Thershold with clinical relevance

$-\rightarrow-$ Moderate high vs. very low

$-\leadsto-\quad$ Moderate high vs. moderate low

Aspects with* indicates the mean score

difference has clinicl relevance

loss, CO constipation, DI diarrhea, FI financial difficulty, EORTC QLQ-OG25 European Organization for Research and Treatment of Cancer Quality of Life Questionnaire-Esophago-Gastric module 25, $O G D Y S$ dysphagia, OGRFX reflux, OGODYN odynophagia, OGPD pain and discomfort, $O G A N X$ anxiety, $O G E O$ eating with others, $O G D M$ dry mouth, OGTA trouble with taste, OGSV trouble with swallowing saliva, $O G C H$ choked when swallowing, $O G C O$ trouble with coughing, $O G S P$ trouble talking, $O G W L$ worry about weight loss, $O G B I$ self-doubt regarding body image

about body image (MSD range -9 to -22 ; Fig. 3; Supplementary Table S2). However, the MSDs for the aspects of dry mouth and trouble with taste were marginal (MSD - 10) and not statistically significant (95\% CI - 21 to 1 and -20 to +0 , respectively; Supplementary Table S2). 
Functional aspects of EORTC QLQ-C30

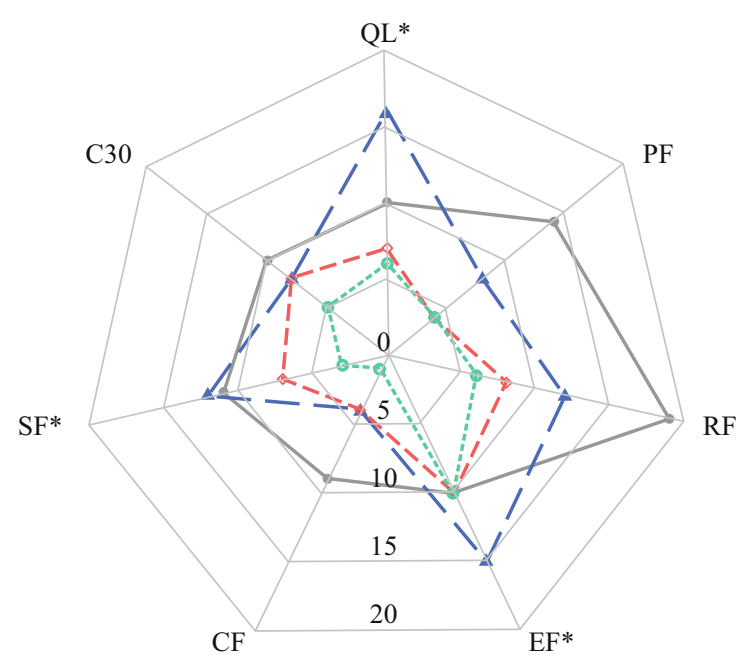

Aspects of EORTC QLQ-OG25

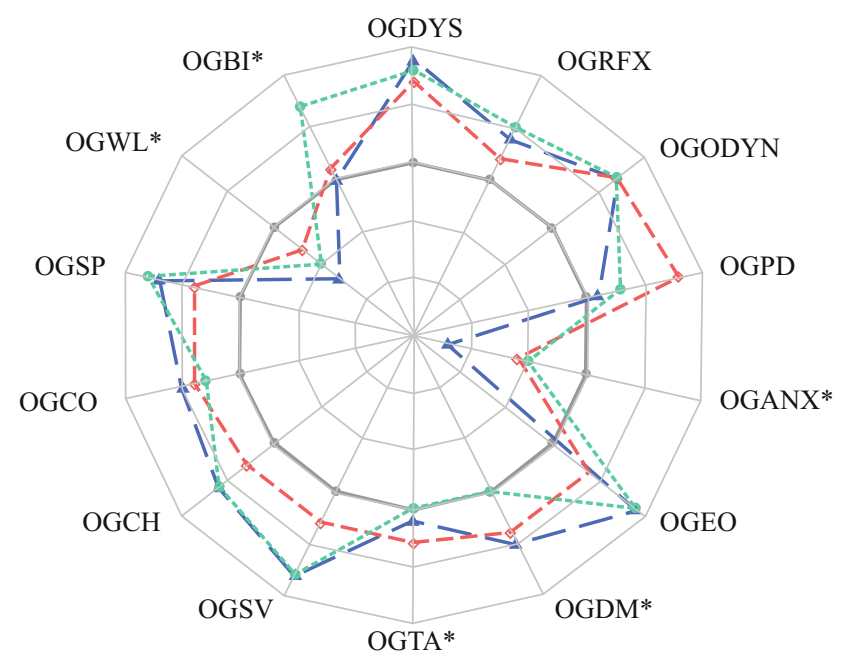

FIG. 3. Mean score differences in health-related quality of life aspects between patients with very high dispositional optimism and lower (very low/moderately low/moderately high) dispositional optimism over the three assessment time points $(1,1.5$, and 2 years after esophageal cancer surgery). EORTC QLQ-C30 European Organization for Research and Treatment of Cancer Quality of Life Questionnaire-Core 30, $Q L$ global quality of life, $P F$ physical function, $R F$ role function, $E F$ emotional function, $C F$ cognitive function, $S F$ social function, C30 EORTC QLQ-C30 summary score, $F A$ fatigue, $N V$ nausea/vomiting, $P A$ pain, $D Y$ dyspnea, $S L$ insomnia,

\section{Time-Varying Predictive Effect of Dispositional Optimism on HRQL}

On the eating difficulty subscale, the predictive effect of dispositional optimism varied over time (interaction effect, $p=0.012$ ). There was no clinically relevant difference

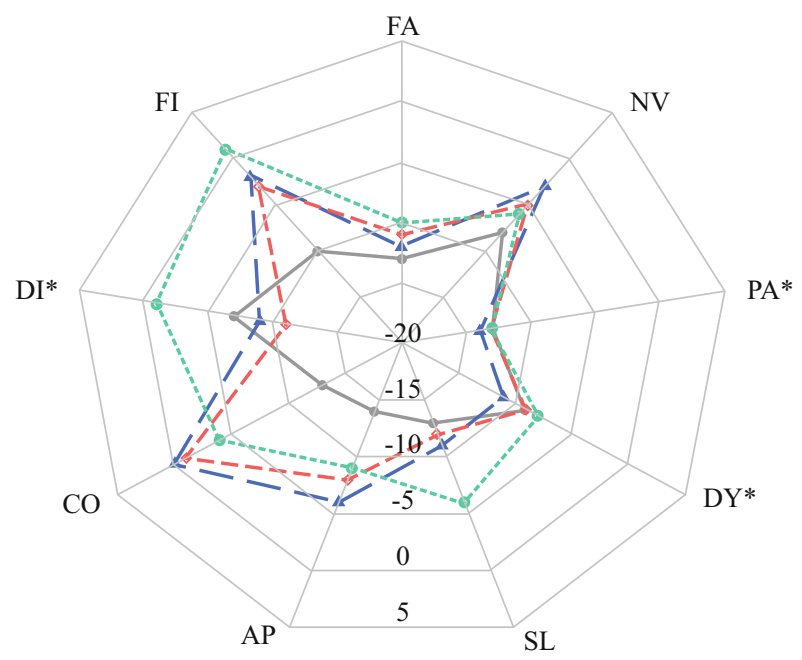

Mean score difference between patients

with different dispositional optisum level

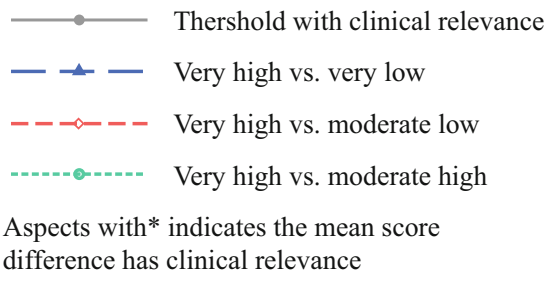

$A P$ appetite loss, $C O$ constipation, $D I$ diarrhea, $F I$ financial difficulty, EORTC QLQ-OG25 European Organization for Research and Treatment of Cancer Quality of Life Questionnaire-EsophagoGastric module 25, OGDYS dysphagia, OGRFX reflux, OGODYN odynophagia, $O G P D$ pain and discomfort, $O G A N X$ anxiety, $O G E O$ eating with others, $O G D M$ dry mouth, OGTA trouble with taste, $O G S V$ trouble with swallowing saliva, $O G C H$ choked when swallowing, $O G C O$ trouble with coughing, $O G S P$ trouble talking, $O G W L$ worry about weight loss, $O G B I$ self-doubt regarding body image

between patients with moderately low and very low dispositional optimism, or between patients with moderately high and lower (very low/moderately low) dispositional optimism (Table 2). However, patients with very high dispositional optimism reported clinically relevant and statistically significant less eating difficulty than patients 
TABLE 2. Mean score difference with $95 \%$ confidence interval (CI) in eating difficulty at 1, 1.5, and 2 years after esophageal cancer surgery between patients with different dispositional optimism levels

\begin{tabular}{|c|c|c|c|c|c|c|c|}
\hline & \multirow{3}{*}{$\begin{array}{l}\text { Time } \\
\text { (years) }\end{array}$} & \multicolumn{6}{|c|}{ Mean score difference $(95 \% \mathrm{CI})$} \\
\hline & & \multirow{2}{*}{$\begin{array}{l}\text { Moderately } \\
\text { low } \\
\text { versus very } \\
\text { low }\end{array}$} & \multicolumn{2}{|c|}{ Moderately high } & \multicolumn{3}{|l|}{ Very high } \\
\hline & & & $\begin{array}{l}\text { versus very } \\
\text { low }\end{array}$ & $\begin{array}{l}\text { versus moderately } \\
\text { low }\end{array}$ & $\begin{array}{l}\text { versus very } \\
\text { low }\end{array}$ & $\begin{array}{l}\text { versus moderately } \\
\text { low }\end{array}$ & $\begin{array}{l}\text { versus moderately } \\
\text { high }\end{array}$ \\
\hline \multirow{3}{*}{$\begin{array}{l}\text { Eating } \\
\quad \text { difficulty }\end{array}$} & 1 & $8(+0,17)$ & $4(-5,12)$ & $-5(-13,4)$ & $-4(-13,4)$ & $-13(-21,-4)$ & $-8(-16,1)$ \\
\hline & 1.5 & $7(-2,16)$ & $7(-2,16)$ & $0(-8,9)$ & $-3(-12,6)$ & $-10(-19,-1)$ & $-10(-19,-2)$ \\
\hline & 2 & $0(-10,10)$ & $\begin{array}{l}-7(-18, \\
3)\end{array}$ & $-7(-17,3)$ & $\begin{array}{l}-11(-21 \\
-1)\end{array}$ & $-11(-21,-1)$ & $-4(-14,7)$ \\
\hline
\end{tabular}

Values marked in bold have both clinical and statistical significance

Mean score difference rounded up to the nearest integer

with lower (very low, moderately low, and moderately high) dispositional optimism, even though the MSDs varied at different time points (Table 2).

\section{DISCUSSION}

This study showed that, compared with patients with lower dispositional optimism, patients with higher dispositional optimism reported better HRQL at 1, 1.5, and 2 years after esophageal cancer surgery, in the aspects of global quality of life, emotional function, social function, anxiety, pain, and body image, which is in line with previous studies conducted among patients with other subtypes of cancer. ${ }^{9-15}$ However, this study further found that dispositional optimism predicted fewer self-reported problems in dyspnea, diarrhea, dry mouth, trouble with taste, and worry about weight loss after esophageal cancer surgery.

The potential mechanisms for these observed associations might be related to coping, goal adjustment, and social support. ${ }^{37-40}$ Previous studies have shown that patients with higher dispositional optimism tend to adopt more effective coping and goal adjustment strategies. ${ }^{37-39}$ When the challenge is controllable, people with higher dispositional optimism tend to make every possible effort to overcome it, but if the challenge is uncontrollable, they also tend to disengage from the unattainable goals and adapt to unfavorable situations more quickly via using emotional acceptance and positive reinterpretation. ${ }^{37,38}$ Therefore, patients with higher dispositional optimism may be more persistent in pursing beneficial lifestyle ${ }^{41}$ such as obeying the special postoperative dietary instructions, quitting smoking, and undertaking more physical exercise, ${ }^{41}$ which might help them reduce the symptoms of diarrhea and eating difficulty, ${ }^{42}$ and relieve their worry about weight loss. In addition, after esophageal cancer diagnosis and surgery, some life goals may become unattainable for patients. Timely disengagements from unrealistic goals and reengagement in new achievable goals can help patients avoid accumulating negative experience and reduce rumination, ${ }^{39}$ which might lead to better emotional function as well as less anxiety and pain. ${ }^{38,39}$ Given that psychological distress is a potential cause of dyspnea, ${ }^{43}$ better emotional function might further help optimistic patients reduce dyspnea symptom. Additionally, more optimistic people are more likely to have higher perceptions of available social support as well as actually receive higher supportiveness from significant others, ${ }^{40}$ thus leading to better social function and less selfdoubt about body image. The combined beneficial effects of high dispositional optimism on the above HRQL aspects might further contribute to better global quality of life.

The observed predictive effect of dispositional optimism on HRQL among patients with esophagectomy for cancer has both clinical and research implications. It may help identify patients at higher risk of suffering from persistently impaired HRQL after esophageal cancer surgery, thus providing tailored follow-up and timely interventions to improve their postoperative HRQL. Moreover, although dispositional optimism is relatively stable, it can be increased via psychological interventions such as cognitive behavior therapy and the Best Possible Self exercise. ${ }^{19}$ The findings of this study suggest that increasing dispositional optimism might be a potential intervention target to improve postoperative HRQL. In addition, given that poor HRQL recovery is associated with higher mortality, ${ }^{44}$ the predictive value of dispositional optimism on HRQL may also imply its potential predictive effect on survival, and future studies on this topic are warranted.

To the best of the authors' knowledge, this is the first study examining the predictive effect of dispositional optimism on HRQL after esophageal cancer surgery. The study reduced confounding bias through adjusting for several potential confounders. HRQL was measured 
comprehensively using both a cancer general questionnaire (EORTC QLQ-C30) and a disease-specific module (EORTC QLQ-OG25). Moreover, the clinical relevance of adjusted MSD was evaluated according to the evidencebased guidelines, and its statistical significance was tested only if it had clinical significance, which not only decreased the risk of chance findings but also ensured the clinical relevance of the results. Additionally, the nationwide population-based longitudinal study design facilitated the generalizability of the findings.

This study also has some limitations. First, because the OSCAR study focuses on patients who have survived for at least 1 year after esophagectomy, we measured dispositional optimism at 1 year post-surgery and not at time of cancer diagnosis or surgery. The observed predictive effect of dispositional optimism on HRQL should be interpreted in light of the assessment time point, even though dispositional optimism remains relatively stable over time and across stressful situations including receiving cancer diagnosis and surgery, ${ }^{45,46}$ and surgical factors were not associated with dispositional optimism based on our data. Second, we categorized patients into four subgroups according to the quartile of LOT-R sum score. Misclassification might happen due to the measurement error in the LOT-R, which can cause potential bias in either direction. ${ }^{47}$ Third, patients with lower dispositional optimism and poor HRQL might be more likely to decline to participate in the study and not answer the follow-up questionnaires, which could make the observed associations underestimated. Last but not least, prediction does not equal causation. The observed association might be due to the effect of unmeasured confounders such as genetic factors, ${ }^{48,49}$ and whether increasing dispositional optimism could improve HRQL needs to be examined by future interventional studies.

In conclusion, this study showed that higher dispositional optimism predicted better HRQL at 1, 1.5, and 2 years post-surgery in several aspects among patients with esophagectomy for cancer. The predictive value of dispositional optimism may help identify high-risk patients with poor HRQL recovery after esophageal cancer surgery, leading to timely and tailored interventions to patients in need, and therefore contribute to the improvement of postoperative HRQL and probably even survival.

ACKNOWLEDGEMENT We thank all patients in the OSCAR study and the patient representatives in our research partnership group for their participation. We thank our OSCAR Project team for their contribution in data collection and validation.

FUNDING Open access funding provided by Karolinska Institutet. The OSCAR study is supported by the Swedish Cancer Society, Region Stockholm (ALF Project), the Cancer Research Funds of
Radiumhemmet, and the Swedish Research Council. Y.L. is supported by the Karolinska Institutet and China Scholarship Council joint program. All funders were not involved in the study design, data collection, data analysis, and manuscript preparation and publication.

DISCLOSURES The authors declare no conflicts of interest.

OPEN ACCESS This article is licensed under a Creative Commons Attribution 4.0 International License, which permits use, sharing, adaptation, distribution and reproduction in any medium or format, as long as you give appropriate credit to the original author(s) and the source, provide a link to the Creative Commons licence, and indicate if changes were made. The images or other third party material in this article are included in the article's Creative Commons licence, unless indicated otherwise in a credit line to the material. If material is not included in the article's Creative Commons licence and your intended use is not permitted by statutory regulation or exceeds the permitted use, you will need to obtain permission directly from the copyright holder. To view a copy of this licence, visit http://creativecommons. org/licenses/by/4.0/.

\section{REFERENCES}

1. Bray F, Ferlay J, Soerjomataram I, Siegel RL, Torre LA, Jemal A. Global cancer statistics 2018: GLOBOCAN estimates of incidence and mortality worldwide for 36 cancers in 185 countries. CA Cancer J Clin. 2018;68(6):394-424.

2. Lagergren P, Avery KN, Hughes R, et al. Health-related quality of life among patients cured by surgery for esophageal cancer. Cancer. 2007;110(3):686-93.

3. Rutegard M, Lagergren J, Rouvelas I, Lindblad M, Blazeby JM, Lagergren P. Population-based study of surgical factors in relation to health-related quality of life after oesophageal cancer resection. Br J Surg. 2008;95(5):592-601.

4. Djarv T, Blazeby JM, Lagergren P. Predictors of postoperative quality of life after esophagectomy for cancer. J Clin Oncol. 2009;27(12):1963-8.

5. Kauppila JH, Xie S, Johar A, Markar SR, Lagergren P. Metaanalysis of health-related quality of life after minimally invasive versus open oesophagectomy for oesophageal cancer. Br J Surg. 2017;104(9):1131-40.

6. Derogar M, Orsini N, Sadr-Azodi O, Lagergren P. Influence of major postoperative complications on health-related quality of life among long-term survivors of esophageal cancer surgery. $J$ Clin Oncol. 2012;30(14):1615-9.

7. Backemar L, Wikman A, Djarv T, Johar A, Lagergren P. Comorbidity after oesophageal cancer surgery and recovery of health-related quality of life. Br J Surg. 2016;103(12):1665-75.

8. Carver CS, Scheier MF, Segerstrom SC. Optimism. Clin Psychol Rev. 2010;30(7):879-89.

9. de Moor JS, de Moor CA, Basen-Engquist K, Kudelka A, Bevers MW, Cohen L. Optimism, distress, health-related quality of life, and change in cancer antigen 125 among patients with ovarian cancer undergoing chemotherapy. Psychosom Med. 2006;68(4):555-62.

10. Colby DA, Shifren K. Optimism, mental health, and quality of life: a study among breast cancer patients. Psychol Health Med. 2013;18(1):10-20.

11. Zenger M, Brix C, Borowski J, Stolzenburg JU, Hinz A. The impact of optimism on anxiety, depression and quality of life in urogenital cancer patients. Psycho-oncology. 2010;19(8):879-86.

12. Allison PJ, Guichard C, Gilain L. A prospective investigation of dispositional optimism as a predictor of health-related quality of 
life in head and neck cancer patients. Qual Life Res. 2000;9(8):951-60.

13. Yoon H, Kim Y, Lim YO, Lee HJ, Choi K. Factors affecting quality of life of older adults with cancer in Korea. Geriatr Gerontol Int. 2015;15(8):983-90.

14. Kung S, Rummans TA, Colligan RC, et al. Association of optimism-pessimism with quality of life in patients with head and neck and thyroid cancers. Mayo Clin Proc. 2006;81(12):1545-52.

15. Finck C, Barradas S, Zenger M, Hinz A. Quality of life in breast cancer patients: Associations with optimism and social support. Int J Clin Health Psychol. 2018;18(1):27-34.

16. Schou I, Ekeberg O, Sandvik L, Hjermstad MJ, Ruland CM. Multiple predictors of health-related quality of life in early stage breast cancer. Data from a year follow-up study compared with the general population. Qual Life Res. 2005;14(8):1813-23.

17. Zaslavsky O, Palgi Y, Rillamas-Sun E, et al. Dispositional optimism and terminal decline in global quality of life. Dev Psychol. 2015;51(6):856-63.

18. Lagergren J, Smyth E, Cunningham D, Lagergren P. Oesophageal cancer. Lancet. 2017;390(10110):2383-96.

19. Malouff JM, Schutte NS. Can psychological interventions increase optimism? A meta-analysis. J Posit Psychol. 2016;12(6):594-604

20. Schandl A, Johar A, Anandavadivelan P, Vikstrom K, Malberg K, Lagergren P. Patient-reported outcomes 1 year after oesophageal cancer surgery. Acta Oncol. 2020;59(6):613-9.

21. Muhonen T, Torkelson EVA. Kortversioner av frågeformulär inom arbets-och hälsopsykologi—om att mäta coping och optimism. Nordisk Psykologi. 2005;57(3):288-97.

22. Scheier MF, Carver CS, Bridges MW. Distinguishing optimism from neuroticism (and trait anxiety, self-mastery, and self-esteem): a reevaluation of the life orientation test. J Pers Soc Psychol. 1994;67(6):1063-78.

23. Liu Y, Pettersson E, Johar A, Lagergren P. Confirmatory factor analysis for Life Orientation Test-Revised among patients with esophageal cancer. PsyArXiv. 2020.

24. Aaronson NK, Ahmedzai S, Bergman B, et al. The European Organization for Research and Treatment of Cancer QLQ-C30: a quality-of-life instrument for use in international clinical trials in oncology. J Natl Cancer Inst. 1993;85(5):365-76.

25. Lagergren P, Fayers $P$, Conroy T, et al. Clinical and psychometric validation of a questionnaire module, the EORTC QLQ-OG25, to assess health-related quality of life in patients with cancer of the oesophagus, the oesophago-gastric junction and the stomach. Eur $J$ Cancer. 2007;43(14):2066-73.

26. Fayers P, Aaronson NK, Bjordal K, Groenvold M, Curran D, Bottomley A. EORTC QLQ-C30 scoring manual. 3rd edn. Brussels: European Organisation for Research and Treatment of Cancer; 2001.

27. Giesinger JM, Kieffer JM, Fayers PM, et al. Replication and validation of higher order models demonstrated that a summary score for the EORTC QLQ-C30 is robust. J Clin Epidemiol. 2016;69:79-88.

28. Brusselaers N, Lagergren J. The Charlson comorbidity index in registry-based research. Methods Inf Med. 2017;56(5):401-6.

29. Glaesmer H, Rief W, Martin A, et al. Psychometric properties and population-based norms of the life orientation test revised (LOT-R). Br J Health Psychol. 2012;17(2):432-45.

30. Schandl AR, Johar A, Malberg K, Lagergren P. Education level and health-related quality of life after oesophageal cancer surgery: a nationwide cohort study. BMJ Open. 2018;8(8):e020702.

31. Anandavadivelan P, Wikman A, Johar A, Lagergren P. Impact of weight loss and eating difficulties on health-related quality of life up to 10 years after oesophagectomy for cancer. $\mathrm{Br} J$ Surg. 2018;105(4):410-8.
32. Vandekerckhove J, Matzke D, Wagenmakers E-J. Model comparison and the principle of parsimony. In: The Oxford handbook of computational and mathematical psychology. New York: Oxford University Press; 2015. p. 300-19.

33. Cocks K, King MT, Velikova G, et al. Evidence-based guidelines for interpreting change scores for the European Organisation for the Research and Treatment of Cancer Quality of Life Questionnaire Core 30. Eur J Cancer. 2012;48(11):1713-21.

34. Cocks K, King MT, Velikova G, Martyn St-James M, Fayers PM, Brown JM. Evidence-based guidelines for determination of sample size and interpretation of the European Organisation for the Research and Treatment of Cancer Quality of Life Questionnaire Core 30. J Clin Oncol. 2011;29(1):89-96.

35. Osoba D, Rodrigues G, Myles J, Zee B, Pater J. Interpreting the significance of changes in health-related quality-of-life scores. $J$ Clin Oncol. 1998;16(1):139-44.

36. King MT. The interpretation of scores from the EORTC quality of life questionnaire QLQ-C30. Qual Life Res. 1996;5(6):555-67.

37. Nes LS, Segerstrom SC. Dispositional optimism and coping: a meta-analytic review. Pers Soc Psychol Rev. 2006;10(3):235-51.

38. Ramirez-Maestre C, Esteve R, Lopez-Martinez AE, SerranoIbanez ER, Ruiz-Parraga GT, Peters M. Goal adjustment and well-being: the role of optimism in patients with chronic pain. Ann Behav Med. 2019;53(7):597-607.

39. Wrosch C, Scheier MF. Personality and quality of life: the importance of optimism and goal adjustment. Qual Life Res. 2003;12(1):59-72.

40. Vollmann M, Antoniw K, Hartung F-M, Renner B. Social support as mediator of the stress buffering effect of optimism: the importance of differentiating the recipients' and providers' perspective. Eur J Pers. 2011;25(2):146-54.

41. Pankalainen M, Fogelholm M, Valve R, et al. Pessimism, diet, and the ability to improve dietary habits: a three-year follow-up study among middle-aged and older Finnish men and women. Nutr J. 2018;17(1):92.

42. Anandavadivelan $P$, Lagergren $P$. Cachexia in patients with oesophageal cancer. Nat Rev Clin Oncol. 2016;13(3):185-98.

43. Neuman A, Gunnbjornsdottir M, Tunsater A, et al. Dyspnea in relation to symptoms of anxiety and depression: a prospective population study. Respir Med. 2006;100(10):1843-9.

44. Djarv T, Metcalfe C, Avery KN, Lagergren P, Blazeby JM. Prognostic value of changes in health-related quality of life scores during curative treatment for esophagogastric cancer. $J$ Clin Oncol. 2010;28(10):1666-70.

45. Bredal IS, Ekeberg $\varnothing$. The stability of dispositional optimism in relation to receiving or not receiving a cancer diagnosis. Psychology. 2016;07(06):806-14.

46. Schou I, Ekeberg O, Sandvik L, Ruland CM. Stability in optimism-pessimism in relation to bad news: a study of women with breast cancer. J Pers Assess. 2005;84(2):148-54.

47. Keogh RH, Strawbridge AD, White I. Correcting for bias due to misclassification when error-prone continuous exposures are misclassified. Epidemiol Methods. 2012;1(1):189-215.

48. Mosing MA, Zietsch BP, Shekar SN, Wright MJ, Martin NG. Genetic and environmental influences on optimism and its relationship to mental and self-rated health: a study of aging twins. Behav Genet. 2009;39(6):597-604.

49. Saphire-Bernstein S, Way BM, Kim HS, Sherman DK, Taylor SE. Oxytocin receptor gene (OXTR) is related to psychological resources. Proc Natl Acad Sci U S A. 2011;108(37):15118-22.

Publisher's Note Springer Nature remains neutral with regard to jurisdictional claims in published maps and institutional affiliations. 